\title{
Design of Virtual Simulation Training System
}

\author{
Hong $\mathrm{Ai}^{1, \mathrm{a}, *}$, Luyuan $\mathrm{He}^{1, \mathrm{~b}}$ \\ ${ }^{1}$ Department of Automation, Harbin University of Science and Technology, No.52 Xuefu Road, \\ Harbin, China \\ a aihong@hrbust.edu.cn, ${ }^{b} 1979429151 @ q q . c o m$ \\ *Corresponding author
}

Keywords: Virtual reality, Simulation, Unity 3D, c \#, 3d Max.

\begin{abstract}
Virtual training is a modern experimental teaching method in recent years. It pays attention to interactive and experimental results of experimental simulation operation. Based on Unity 3D, it designed a virtual simulation training system for measuring laser wavelengths by Michelson interferometer. Virtual simulation experiment is used to explore the basic principles and methods of operation. Try to use 3DMax software to design laboratory and laboratory equipment model. Through Unity3D software builds the experimental environment and operation of the logical relationship test algorithm. Use program to achieve shader optical visualization. It can enhance the practice of students' practical ability to reflect the new generation of experimental teaching of the open and comprehensive features.
\end{abstract}

\section{Introduction}

The virtual simulation training system simulates the development of $3 \mathrm{D}$ digital content based on the specific subject content and simulates the training environment with $3 \mathrm{D}$ virtual environment or 3D stereoscopic display device so that teachers and students can obtain the same or similar training experience in real world. It is a new type of teaching model and the market is broad ${ }^{[1]}$. In order to meet needs in the Internet education, this paper studies the virtual simulation system of 3D simulation based on the background of laser measurement. It shows the multi-information fusion interactive three-dimensional dynamic scene, to a certain extent, reflects the VR education intuitive and immersive ${ }^{[2]}$.

\section{Unity3D engine}

Unity3D is a Danish company launched for a game development, three-dimensional simulation, and cross-platform development of the three-dimensional engine. The Unity3D editor runs on Windows and Mac. It is a game product and software based on Android, Windows, Wii, OSX and iOS platform released, as shown in Figure 1. Unity engine achieves a multi-platform publishing and information exchange ${ }^{[3]}$. The development environment of Unity3D is friendly and visual. It supports the $\mathrm{c} \#$ scripting language and the model created by $3 \mathrm{dsMax}^{[4-5]}$. In this article, unity engine model rendering technology is used for different materials and different attributes of the 
equipment in the virtual training environment to achieve efficient 3D view display. Unity operation interface shown in Figure 2.

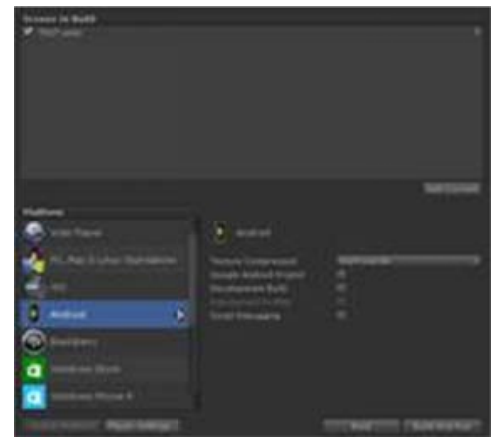

Figure 1 Multi-platform release

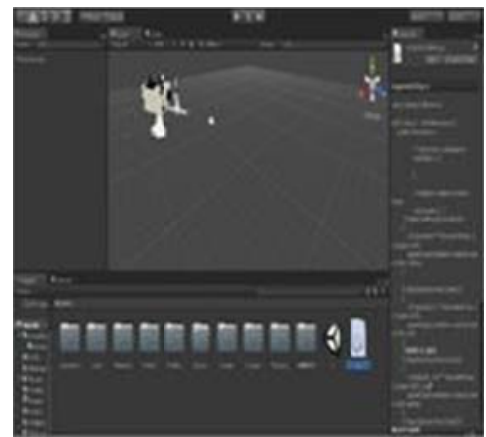

Figure 2 Unity operation interface

\section{Virtual Simulation Training System Workflow}

As shown in Figure 3:

(1) Through the selected virtual experiment, remove the virtual instrument, in the fixed virtual experiment, and functional and model module segmentation. After the completion of the instrument function, the functions are reassembled to improve the integration of the virtual instrument.

(2) The reassembled virtual instrument must be grouped.

(3) After the group of virtual instruments, there must be similar to the formation of virtual experiments, the formation of virtual experiments is to verify the key to the experimental system.

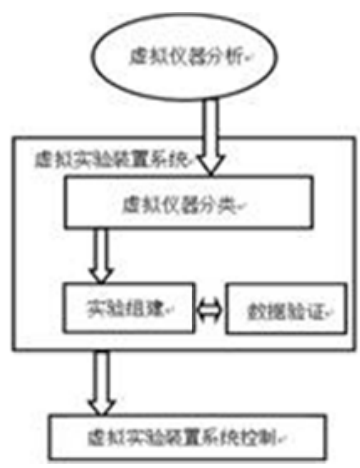

Figure 3 Virtual simulation system

\section{3D Max Model Processing}

3D Max design of the virtual training system in the three-dimensional model can be modified with materials such as light and shadow. 3D Max can adjust the proportion of virtual instruments, the coordinate system centre point and the centre of mass position. Adjust the proportion and position of the three-dimensional structure to achieve the same as the real experiment equipment. This virtual experiment instrument model can improve the authenticity of the experiment. As shown in Figure 4, the model's material rendering process includes: texture, reflection, and highlight. The figures before and after rendering are shown in Figure 5 and Figure 6. 


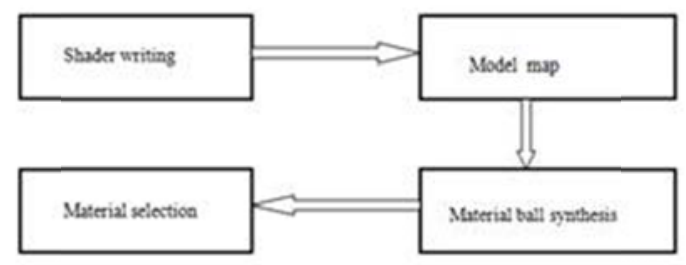

Figure 4 Model rendering process

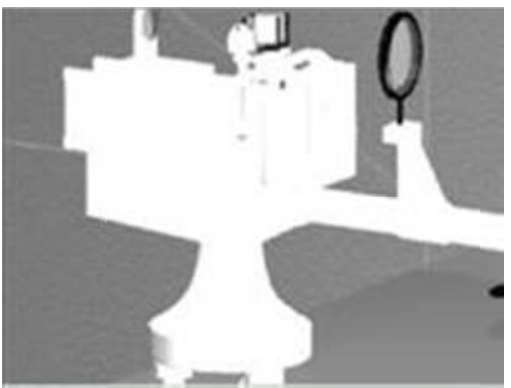

Figure 5 Before rendering the model

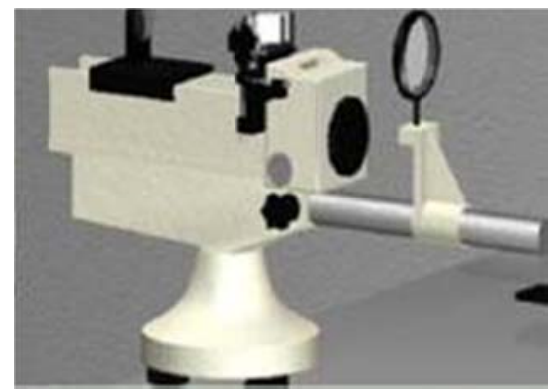

Figure 6 After the model is rendered

\section{Experimental Theory}

The experimental principle of the Michelson interferometer is shown in Figure 7, assuming that the thickness of the gap air layer at the Newton ring interference is $n$, and then the phase difference of the two coherent lights is equal to the light intensity difference. If the thickness of the gap film of M1 and M2' is d, the angle of incidence and the angle of incidence of 45 degrees on the half mirror surface are z, and the angle between the incident light of S1' and S2' and the lens E is also z, d is the light source S1' And S2' distance of 1/2. Optical path difference formula $\Delta$ :

$$
\Delta=2 \mathrm{~d} \cos (\mathrm{z})
$$

When M1 and M2' distance d unchanged with all the same incident angle of the incident light, have the same $\Delta$, so $\mathrm{S}^{\prime}$ and $\mathrm{S} 2$ ' issued the same inclination of the light polymerization in the $\mathrm{E}$ reflection plane and then form an equidistant interference pattern, the thickness of different of the stripes for the bright and dark color of the concentric circles. M-level ring formed conditions, the laser wavelength $\lambda$ is:

$$
\lambda=2 \mathrm{~d} \cos (\mathrm{z}) / \mathrm{m}
$$

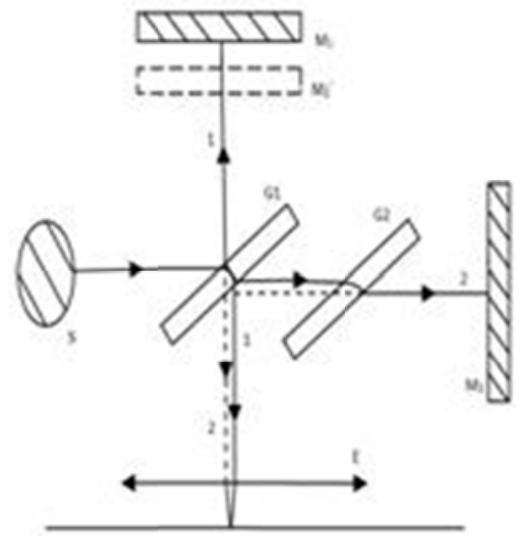

Figure 7 Experimental principle 


\subsection{Analysis of concentric circle fringe algorithm}

Assume that the distance from the lens to the receiving screen $\mathrm{A}$ is $\mathrm{M}$, when the incident angle is z. Radius of interference ring $r$ :

$$
\operatorname{rcos}(z)=M /\left(\sqrt{r^{2}+M^{2}}\right)
$$

The corresponding $\Delta$ is indicated by the convergence of the light beam 1 and the light beam 2 on the receiving screen. The phase difference can be written as:

$$
\emptyset=4 \pi \mathrm{d} / \lambda * \mathrm{M} /\left(\sqrt{\frac{1}{2}} \emptyset\right)
$$

The light intensity of the light beam 1 and the light beam 2 to the receiving screen $\mathrm{A}$ is $\mathrm{I}$ ', and the light intensity I after the polymerization is show in formula(5):

$$
\mathrm{I}=4 \mathrm{I}^{\prime} *\left(\sqrt{\frac{1}{2} \emptyset}\right)
$$

Concentric ring fringe law: when the fringes generated in the focal plane A, the corresponding experimental phenomenon will have a circle of concentric rings, each concentric ring corresponds to a radius $\mathrm{r}$, and corresponds to an angle of incidence $\mathrm{z}$. And each incident angle $\mathrm{z}$ corresponds to $\Delta$ and $\emptyset$.

\section{2 concentric circles drawn}

As for the formula(5), determining the constant distance $\mathrm{M}$ and $\mathrm{d}$ in the mixed light intensity I for the adjustment of the experimental variables, selecting the different values of the interference experiment, making use of shader programming to simulate the real light. And the light color, the shading effect is displayed in the form of an image in the computer. Define a method to deal with stripes to draw concentric circles by c\#.

Striped color design:

(1) Mixed light intensity I quantized between $0-1$, stripes and color values rg b corresponding.

(2) Making use of colour processing color function to distinguish between different radius concentric fringe colours.

(3) As shown in Figure 8, after the stripes are drawn, the setting $d$ is adjustable by means of the Michelson interferometer's coarse adjustment knob and the fine adjustment knob to rotate the angle to show the change of $d$ on the Michelson interferometer.

\section{Set up virtual training environment}

Different wavelengths corresponding to different visible light, as shown in Figure 9, c\# programming assign the corresponding material ball to the light to achieve different wavelengths of choice and measurement, encapsulated it into a separate class assigning to the $3 \mathrm{D}$ virtual instrument model. In this way, the model has the performance of the corresponding optical instruments. 


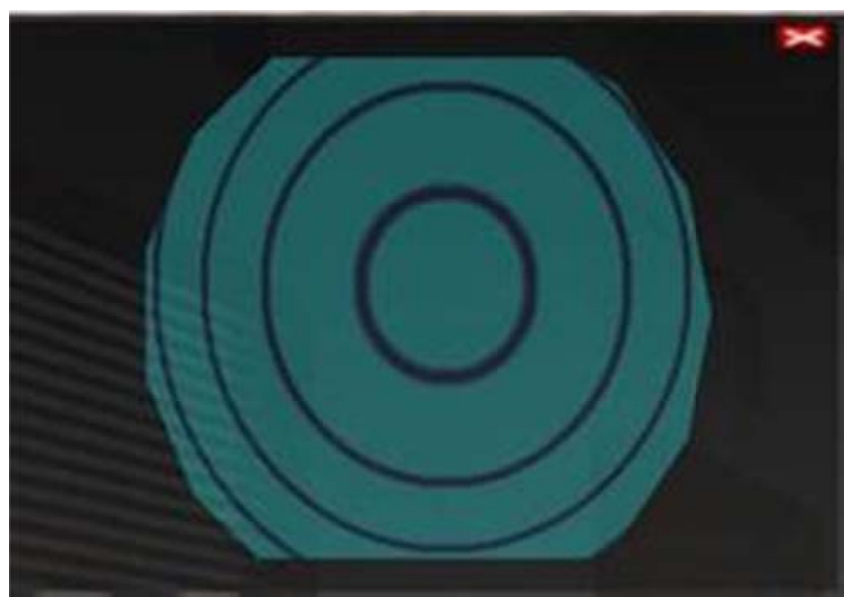

Figure 8 Experimental stripes effect

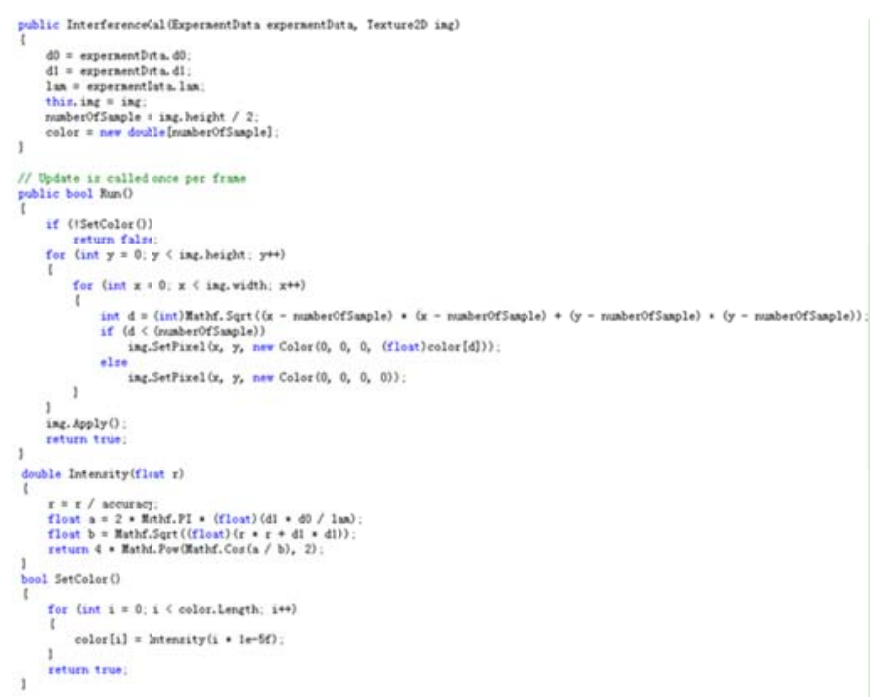

Figure 9 C\# program

The model is derived from 3DMax, the real image as its texture mapping to form a 3D virtual scene.

(1) Pay attention to the establishment of the laboratory interior structure and the map modifying the floor ceiling. Add virtual laboratory internal modifier including gray metal test bed, Michelson interferometer, ordinary seats, projectors and projection panels, aperture diaphragm, all colored laser lasers.

(2) Unify the number of maps and the internal decorations of the experiment to manage it for easier replacement and use.

(3) After experimental environment to be carried out, it needs the light operation so that the simulation results can become more realistic. The virtual lab is shown in Figure 10.

\section{Virtual operation logic relationship}

Determining experimental models such as Michelson interferometers, general seats, projectors and projectors, aperture apertures, all colored laser lasers. Initializing each model location and roaming camera location. All using the first person perspective in the entire virtual experiment show the sense of immersion of VR. The logic flow is shown in Figure 11. 


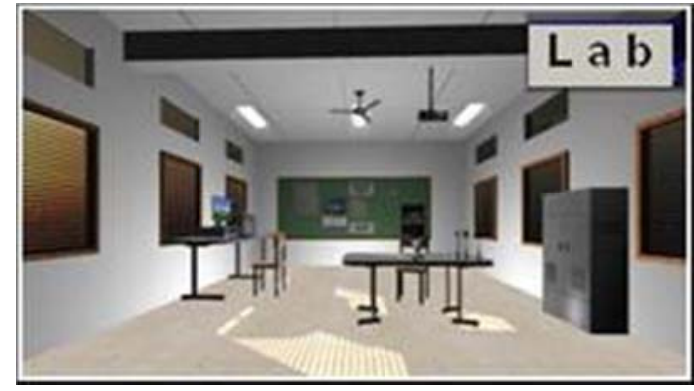

Figure 10 Virtual training environment

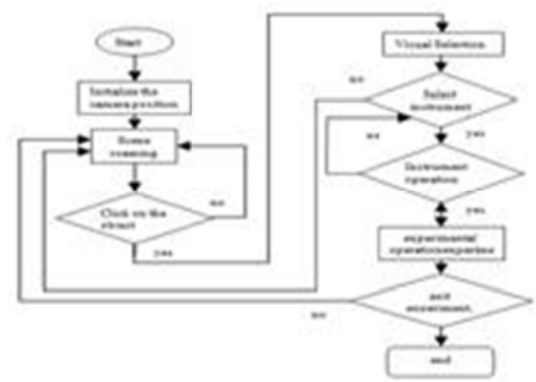

Figure 11 Experimental logic relationship

\section{Virtual training operation}

Design third-party plug-in interface and button function, enter the software after the experimental interface shown in Figure 12. Through the mouse click on the experimental seat, adjust the experimental equipment, ready to operate.

Step1: enter the experimental position, set the measured colored light to adjust the laser transmitter emission wavelength shown in Figure 13.

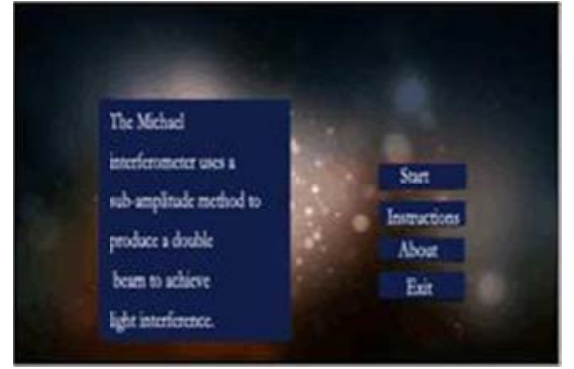

Figure 12 Experimental interface

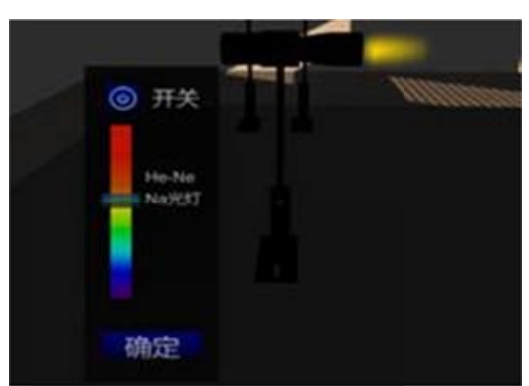

Figure 13 Laser emitter

Step 2: Set the aperture diaphragm and short focus lens to reduce the radius of the transmitted laser beam and diffuse the laser beam, as shown in Figure 14.

Step 3: After setting up the virtual instrument, perform the virtual experiment operation. The Michelson interferometer is placed on the laser wavelength measurement and the concentric circles are sucked or released by adjusting the fine focal length helix. When 30 concentric circles are sucked out or discharged, the experimental data is recorded. Make several sets of experimental record data, and then experimental verification is shown in Figure 15, Figure 16.

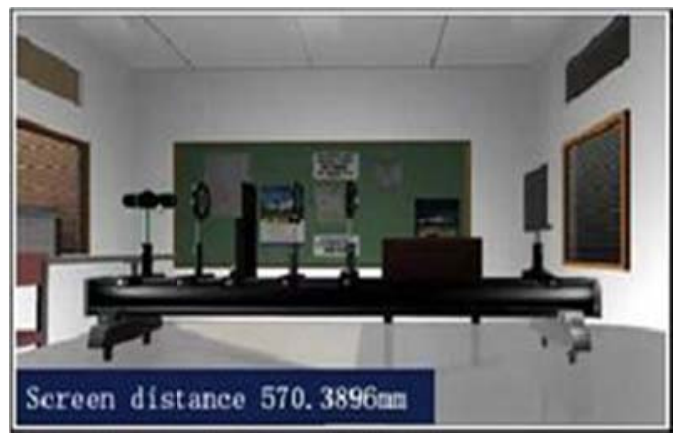

Figure 14 Virtual instrument

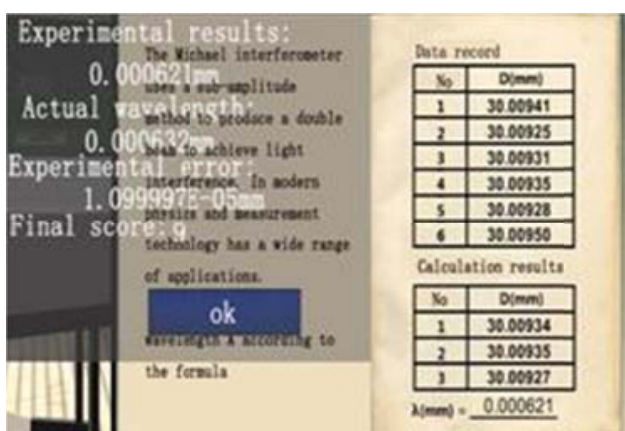

Figure 15 Experimental data records 


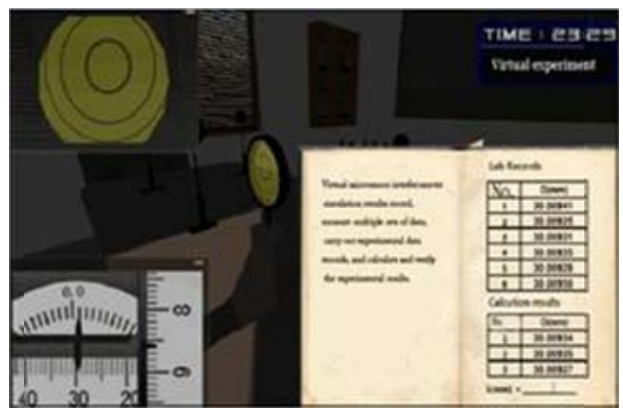

Figure 16 Michael interferometer operation and measurement of numerical records

\section{Conclusions}

Based on Unity3D game engine development of virtual simulation, the training system can be cross-platform released, it is closed to the real 3D experimental environment and user-friendly user interface. Unity3D in the three-dimensional simulation model and the real environment achieve a perfect fusion. In this paper, through analysis of the virtual simulation experiment Michelson interferometer measurement of laser wavelength, virtual simulation training system in teaching not only can reduce the real experiment due to the damage of limited equipment and improper operation, but also improve student autonomy, making use of network to expand the exchange between people, reflecting the characteristics of VR education and fun.

\section{Acknowledgements}

*This work is supported by Ministry of Education Humanities and Sociall Sciences engineering science and technology talents special (15JDGC020).

\section{References}

[1] Wan Huagen, Gao Shuming, Peng Qunsheng. VDVAS: an integrated virtual design and virtual assembly environment[J]. Chinese Journal of Computer \& Graphics, 2002, 7(1): 27-35.

[2] Chen-Yuan Chen, Bih-Yaw Shih, Shih-Hsien Yu.Disaster prevention and reduction for exploring teachers' technology acceptance using a virtual reality system and partial least squares techniques[J].Natural Hazards, 2012, 62(3):1217-1231

[3] Johnston R, Weiss P.. Analysis of virtual reality technology applied in education[J].Minimally Invasive Therapy \& Allied Technologies, 2009, 6(2):126-127

[4] Yang Jiancheng. Research on Virtual Simulation Experiment Center Construction Practice and Resource Sharing Management Mechanism-Taking Mechanical Foundation and Textile Equipment Experiment Center as an Example[A]. Singapore Management University. Proceedings of the 1st International Conference on Education, Management Science and Economics (ICEMSE 2016)[C].Singapore Management University:,2016:5.

[5] Xiang Ming. A Review of Research on Virtual Simulation in Education and Teaching[A]. Singapore Management University.Proceedings of the 1st International Conference on Education, Management Science and Economics (ICEMSE 2016)[C].Singapore Management University:2016:4. 